\title{
Response of antimicrobial nitrofurazone-degrading biocathode communities to different cathode potentials
}

\author{
Deyong Kong a,b , Hui Yun ${ }^{c}$, Dan Cui ${ }^{\text {c }}$, Mengyuan Qi ${ }^{\mathrm{b}}$, Chunyan Shao ${ }^{\mathrm{a}}$, Dichen Cui ${ }^{\mathrm{a}}$, Nanqi Ren ${ }^{\mathrm{b}}$, \\ Bin Liang ${ }^{\mathrm{c}, *}$, Aijie Wang ${ }^{\mathrm{b}, \mathrm{c}}$ \\ a Shenyang Academy of Environmental Sciences, Shenyang 110167, China \\ ${ }^{\mathrm{b}}$ State Key Laboratory of Urban Water Resource and Environment, Harbin Institute of Technology, Harbin 150090, China \\ ${ }^{\mathrm{c}}$ Key Laboratory of Environmental Biotechnology, Research Center for Eco-Environmental Sciences, Chinese Academy of Sciences, Beijing 100085, China
}

\section{H I G H L I G H T S}

- NFZ bioelectrodegradation degree was related to different cathode potentials.

- Different cathode potentials enriched specific biocathode communities respectively.

- Dominant genera possess nitroaromatics reduction and electron transfer functions.

- Higher potential modes enriched a Gram negative nitroaromatic reducer Klebsiella.

- Lower potential mode enriched a Gram positive nitroaromatic reducer Enterococcus.

\section{A R T I C L E I N F O}

Article history:

Received 20 April 2017

Received in revised form 6 June 2017

Accepted 10 June 2017

Available online 13 June 2017

\section{Keywords:}

Nitrofurans nitrofurazone (NFZ)

NFZ bioelectrodegradation

Biocathode community structure

Cathode potential regulation

\begin{abstract}
A B S T R A C T
Bioelectrodegradation of various organic pollutants has been extensively studied. However, whether different cathode potentials could alter the antimicrobial-degrading biocathode community structure and composition remain poorly understood. Here, the microbial community structure and composition of the nitrofurans nitrofurazone (NFZ) degrading biocathode in response to different cathode potentials $(-0.45 \pm 0.01,-0.65 \pm 0.01$ and $-0.86 \pm 0.05 \mathrm{~V}$ vs standard hydrogen electrode, with applied cell voltages of $0.2,0.5$ and $0.8 \mathrm{~V}$, respectively) were investigated. The bioelectrodegradation efficiency and degree of NFZ were highly related to different cathode potentials. The 0.2 and $0.5 \mathrm{~V}$ performed biocathode communities were similar but significantly differed from those of the $0.8 \mathrm{~V}$ and open circuit biofilms. The bacteria possessing functions of nitroaromatics reduction and electrons transfer (e.g. Klebsiella, Enterococcus, Citrobacter and Desulfovibrio) were selectively enriched in different biocathode communities. This study offers new insights into the ecological response of antimicrobial-degrading biocathode communities to different cathode potentials.
\end{abstract}

(c) 2017 Elsevier Ltd. All rights reserved.

\section{Introduction}

Antimicrobial agents have led to serious pollution of various environmental settings globally, including surface water and groundwater, estuary sediment and soils, for excessively abused in humans, domestic animals, and aquaculture (Justino et al., 2016; Van Boeckel et al., 2015; Yun et al., 2017b; Zhang et al., 2015). Nitrofurazone (5-nitro-2-furaldehyde semicarbazone, NFZ) is a broad-spectrum antimicrobial agent that belongs to nitrofurans. Due to its potential carcinogenicity, genotoxicity and mutagenicity to human health, the European Union and U.S. Food and

\footnotetext{
* Corresponding author.

E-mail addresses: liangbin1214@163.com, binliang@rcees.ac.cn (B. Liang).
}

Drug Administration have banned the utility of NFZ in foodproducing animals. However, in some developing countries including China, it is still widely used in the livestock and fishery aquaculture owing to its low production cost, easy availability and high efficiency (Hong et al., 2015; Kwon, 2017; Vass et al., 2008). As a result, NFZ residues and its toxic biological metabolite semicarbazide are often detected in diverse aquatic species and products as well as aquaculture pond water and sediments (Vass et al., 2008; Yu et al., 2013). In order to eliminate the ecological impact of NFZ on water environment, the efficient degradation of NFZ and elimination of its antibacterial activity are very important during livestock wastewater treatment and aquaculture pond water remediation. Thus, NFZ was chosen as the model nitrofurans in this study. 
Bioelectrochemical systems (BES), employing electrochemically active microorganisms to catalyze oxidative or reductive reactions in the anode or cathode respectively, have attracted a considerable amount of attentions in recent decades (Pandey et al., 2016; Pant et al., 2010; Wang et al., 2015; Yun et al., 2017a), especially its merits on the bioelectrodegradation of various organic pollutants including nitro-, azo-, halo-aromatics and antimicrobial agents based on the microbial electrode-respiration process (Cui et al., 2014, 2016; Feng et al., 2015; Gao et al., 2016; Jiang et al., 2016a; Li et al., 2016; Liang et al., 2013; Sun et al., 2013; Wang et al., 2011, 2016; Zhang et al., 2017). Generally, operational factors could impact the catalytic efficiency and functioning stability of abiotic cathode/biocathode reactors (e.g. temperature, $\mathrm{pH}$, buffer solution type and concentration, targeted pollutants concentration and analogue, co-substrate addition, and electrode potential) (Jiang et al., 2016a,b; Kong et al., 2014, 2015b; Liang et al., 2014, 2016; Shen et al., 2013; Yun et al., 2016, 2017a). Our previous studies have indicated that nitrofuran derivatives NFZ and furazolidone could be efficiently reduced by the abiotic cathode under different cathode potentials $(-0.15$ to $-1.25 \mathrm{~V}$ vs standard hydrogen electrode, SHE). Moreover, different cathode potentials significantly affected the NFZ and furazolidone degradation efficiency and the elimination of degradation products (Kong et al., 2015a,b). However, whether the acclimated biocathode communities could enhance NFZ degradation has not yet been reported. Our recent studies have shown that the polarity inversion (decrease electrode potential from -0.2 to -0.45 or $-0.40 \mathrm{~V}$ ) could significantly alter the bioanode community structure and composition (Yun et al., $2016,2017 a)$. Interestingly, a previous study has shown that different anode potentials (from -0.25 to $0.81 \mathrm{~V}$ vs SHE) did not affect bioanode community structure and composition (Zhu et al., 2014). However, whether the lowered cathode potentials (below $-0.45 \mathrm{~V}$ vs SHE) could alter the functional biocathode community structure and composition and whether different cathode potentials could selectively enrich distinct electroactive nitroaromatic reducers remain poorly understood.

This study investigated the effect of different cathode potentials on the bioelectrodegradation efficiency and degree of NFZ in a dual-chamber BES with biocathode. The NFZ bioelectrodegradation characterization, products formation dynamics, and biocathode communities diversity, structure and composition were studied. The objectives of this study were to (I) determine the impact of different cathode potentials on the bioelectrodegradation of NFZ, formation and further elimination of corresponding degradation products and (II) elucidate the ecological response of the NFZdegrading biocathode communities to different cathode potentials. Our results indicated that the bioelectrodegradation efficiency and degree of NFZ were highly related to different cathode potentials, and lower and higher cathode potentials enriched different electroactive nitroaromatic reducers respectively.

\section{Materials and methods}

\subsection{BES reactor setup}

The BES reactors were constructed by assembling two equalsized Lexan plates $\left(4 \times 4 \times 3 \mathrm{~cm}^{3}\right)$ with a cylindrical cavity $(3 \mathrm{~cm}$ in diameter and $4 \mathrm{~cm}$ in length) and two equal-sized Lexan plates $\left(4 \times 4 \times 1 \mathrm{~cm}^{3}\right)$ as outside baffles and separated by a cation exchange membrane (Ultrex CMI-7000, Membranes International, Ringwood, NJ, U.S.) as described elsewhere (Kong et al., 2015a). The internal volume of each chamber was $28 \mathrm{~mL}$. Graphite fiber brush $(2.5 \mathrm{~cm}$ in diameter and $2.5 \mathrm{~cm}$ in length, TOHO TENAX Co., Ltd., Japan) was used as both anode and cathode. A $10 \Omega$ resistor with the external power $(0.5 \mathrm{~V})$ in series was employed for the connection. A saturated calomel reference electrode (SCE, $0.247 \mathrm{~V}$ vs SHE, model-217, Shanghai Precise. Sci. Instrument Co., Ltd., China) was inserted into the cathode chamber. All the potential data showed herein were against SHE. The open circuit control was also carried out in the same reactor by directly inoculating NFZ-reducing consortium under non-electrochemical anaerobic condition (the external power source and the resistor were disconnected).

\subsection{Microbial inoculum and BES tests}

Three biocathodes were parallel established with applied cell voltage of $0.5 \mathrm{~V}$ and glucose (additional carbon and electron sources) supply. In order to determine the effect of different cathode potentials on the bioelectrodegradation efficiency and degree of NFZ, two of the three established biocathodes were switched to supply the additional voltages of 0.2 and $0.8 \mathrm{~V}$. While one biocathode still maintained $0.5 \mathrm{~V}$ supply. An open circuit and a 0.8 V-supplied abiotic cathode BES reactors both worked as controls to assess the contribution of pure microbial or electrochemical degradation of NFZ respectively. These reactors were operated for 6 fed-batch cycles ( $48 \mathrm{~h}$ for each cycle). Then the three biocathode BESs were run without the additional electron donor glucose (with $10 \mathrm{mM} \mathrm{NaHCO}_{3}$ instead) for another 3 cycles. For the glucose-fed biocathode mode, NFZ (50 mg/L), glucose $(600 \mathrm{mg} / \mathrm{L})$ and cathode were the cathodic electron acceptor, intracellular and extracellular electron donors for cathodophilic microbes, respectively. For the $\mathrm{NaHCO}_{3}$-fed biocathode mode, inorganic $\mathrm{NaHCO}_{3}$ and electrode served as carbon source and electron donor for cathodophilic microbes, respectively. All tests were carried out at room temperature $\left(23 \pm 3^{\circ} \mathrm{C}\right)$.

Concretely, anodic and cathodic biofilms were enriched by inoculating municipal sludge from a wastewater treatment plant (Harbin, China) and acclimated NFZ-reducing consortium, respectively as described elsewhere (Liang et al., 2013; Wang et al., 2011). Cathode biofilms were established by inoculating NFZ-reducing consortium three times into the BES reactor. For the NFZ-reducing consortium enrichment, wastewater and sludge samples (about $5 \mathrm{~mL}$ ) from a wastewater treatment plant (Shijiazhuang, China) were mixed with $70 \mathrm{~mL}$ anaerobic medium, which was prepared by boiling and purged with $\mathrm{N}_{2}$ gas (99.9\% purity) for $15 \mathrm{~min}$. The medium composition was described previously but amended with NFZ (50 mg/L) and glucose (600 mg/L) (Wang et al., 2011). The mixed culture was incubated in a rotary shaker at $160 \mathrm{rpm}$ and $25{ }^{\circ} \mathrm{C}$ for $4 \mathrm{~d}$, and then was transferred to a fresh medium ( $70 \mathrm{~mL}$ ) with $10 \%$ dilution for another $4 \mathrm{~d}$. This procedure was repeated six times. Finally, the NFZ acclimated consortium is capable of completely reducing NFZ to the antibacterial inactive products within $72 \mathrm{~h}$.

\subsection{Chemicals and analytical methods}

NFZ (98\% purity) was purchased from Aladdin (Shanghai, China). HPLC grade methanol was purchased from Sigma-Aldrich (St. Louis, MO, USA). All other chemicals used were of analytical grade.

All samples taken from the cathode chamber within $72 \mathrm{~h}$ were filtered through $0.22 \mu \mathrm{m}$ filters (Shanghai XingYa Purification Material Co., China). The concentrations of NFZ and its three main products, [(5-hydroxyamino-2-furyl)-methyl]-hydrazinecarboxa mide (AMH), [(5-amino-2-furyl)-methylene]-hydrazinecarboxa mide (AMN) and $\mathrm{N}-\mathrm{N}$ fraction product (5-nitro-2-furyl) methanamine (NFF) were measured using a reverse-phase HPLC system with a UV detector (model e2695, Waters Co., Milford, MA, USA) (Kong et al., 2015a). The NFZ reduction products were identified by a HPLC-QTOFMS as described recently (Kong et al., 2015a). 
The standard samples for NFZ degradation products NFF, AMN, and AMH are not available commercially. Therefore, we used the HPLC peak area to describe the concentration or mass change of these products during NFZ degradation. The rate constant of NFZ reduction $\left(k_{\mathrm{NFZ}}\right)$ was calculated by fitting the data to apparent first-order reaction models: $C=C_{0} \mathrm{e}^{-k t}$ and $C=C_{0}\left(1-\mathrm{e}^{-k t}\right)$, where $C$ represents the NFZ $(\mathrm{mg} / \mathrm{L})$ concentration at time $t(\mathrm{~h})$ and $C_{0}$ is the initial NFZ concentration $(50 \mathrm{mg} / \mathrm{L}$ ). The electric current (I) was calculated by the Ohm's law. NFZ reduction efficiency $\left(\mathrm{E}_{\mathrm{r}-\mathrm{NFZ}}\right.$ \% $)$ was evaluated based on the concentration difference between influent and effluent. Differences between treatments were statistically analyzed by the two-tailed unpaired $t$-test.

\subsection{Electrochemical and morphological analysis}

To characterize the bioelectrochemical activity of biocathode communities under different cathode potentials, CV analysis was performed using an electrochemical workstation (model-660D, CH Instruments Inc., Austin, TX, U.S.) as described previously (Liang et al., 2013). The electrochemical impedance spectroscopy (EIS) test was carried out over a frequency range of $0.01-10^{5} \mathrm{~Hz}$ and amplitude of $5 \mathrm{mV}$ (model-660D, CH Instruments Inc., Austin, TX, U.S.) for the analysis of the polarization internal resistance of biocathode communities under different cathode potentials. In order to exclude the additional effect of glucose on the biocathodic metabolism, in the $\mathrm{CV}$ and EIS experiments, inorganic $\mathrm{NaHCO}_{3}$ was selected as carbon source and electrode as electron donor. For the uniformity and stability of the experimental conditions, the second cycle of the scanned cyclic voltammograms were shown. The concentration of NFZ and $\mathrm{NaHCO}_{3}$ were $50 \mathrm{mg} / \mathrm{L}$ and $10 \mathrm{mM}$, respectively in the CV and EIS analysis at $25^{\circ} \mathrm{C}$. The cathodic biofilms were observed by scanning electron microscopy (SEM) (HELIOS Nanolab 600i, Hillsboro, OR, USA) as described elsewhere (Wang et al., 2012).

\section{5. $16 S$ rRNA gene sequencing and data analysis}

A detailed description of DNA extraction, DNA purity and quantity determination, PCR amplification, 16S rRNA gene Illumina MiSeq sequencing and data analysis was provided in our previous study (Cheng et al., 2015; Liang et al., 2016). The raw data from 16S rRNA gene sequencing was analyzed according to a recent study (Qu et al., 2015). Microbial diversity indices including Chao1 value, richness (identified OTU numbers in each sample), ShannonWeaver index $(H)$, Simpson reciprocal $(1 / D)$ and Pielou evenness were calculated as described recently (Liang et al., 2016; Schloss et al., 2009). For indices, higher number represents higher diversity. Hierarchical clustering analysis was performed using CLUSTER v3.0 and visualized by TREEVIEW.

\section{Results and discussion}

\subsection{NFZ-reducing consortium capability}

After the 6th transfer, the NFZ-reducing capability of the acclimated consortium was determined. CV analysis preliminarily indicated that almost no reductive current was recorded for the enrichment within $72 \mathrm{~h}$, which was quite close to the cyclic voltammogram of PBS control. In contrast, NFZ containing medium ( $50 \mathrm{mg} / \mathrm{L}$ ) showed three obvious reduction peaks those were consistent with our previous study (Kong et al., 2015a). CV result indirectly showed the added NFZ could be efficiently reduced by the acclimated NFZ-reducing consortium (Fig. S1A). Further HPLC analysis demonstrated the acclimated consortium is able to reduce NFZ $(50 \mathrm{mg} / \mathrm{L})$ to three main products of NFZ reduction (Fig. S1B). Inter- estingly, these three main products (retention time at $3.2 \mathrm{~min}$, $3.5 \mathrm{~min}$ and $3.6 \mathrm{~min}$ respectively) were the same as the NFZ reduction products from the pure electrochemical process. They were preliminarily identified as NFF, AMN and AMH (Kong et al., 2015a). These results suggest that microbial nitroreductase and $\mathrm{N}-\mathrm{N}$ bond reductase catalyze the reduction of NFZ.

\subsection{Bioelectrodegradation of NFZ under different operational conditions}

After inoculating the NFZ-reducing consortium for three times, cathodic biofilms were established after $196 \mathrm{~h}$. During the biofilm establishment process, the cathode potential kept between -0.75 and $-0.80 \mathrm{~V}$ with the applied cell voltage of $0.5 \mathrm{~V}$ and currents of the three reactors obviously increased over the first cycle. After 3 cycles, the current increased from approximately 0.2 to $0.3 \mathrm{~mA}$, suggesting that the electroactive NFZ-degrading cathodic biofilm was preliminarily established (Fig. 1a). The current and cathode potential of the established biocathodes changed obviously after applying different cell voltages $(0.2,0.5$ and $0.8 \mathrm{~V})$. As a result, the $0.2,0.5$ and $0.8 \mathrm{~V}$ groups achieved the mean cathode potential of $-0.45 \pm 0.01,-0.65 \pm 0.01$ and $-0.86 \pm 0.05 \mathrm{~V}$ vs SHE, respectively. The $0.8 \mathrm{~V}$-supplied biocathode showed the highest current among the three biocathode groups, indicating that NFZ could be more efficiently reduced under a lower cathode potential (Fig. 1b). With $\mathrm{NaHCO}_{3}$ and cathode as carbon source and electron donor respectively, the variation trends of cathode potential and current were similar to those with glucose as carbon source and additional electron donor. The higher applied voltage resulted in lower cathode potential and higher corresponding reaction current (Fig. 1c). This indicated that the use of $\mathrm{NaHCO}_{3}$, an inorganic carbon source instead of glucose, did not change the reactor performance significantly.

When the applied cell voltage was $0.2 \mathrm{~V}$, the reduction rate constant $k$ and reduction efficiency at $1 \mathrm{~h}\left(\mathrm{E}_{\mathrm{r} 1 \mathrm{~h}}\right)$ of NFZ were $0.677 \pm 0.069 \mathrm{~h}^{-1}$ and $42.25 \pm 1.35 \%$ with glucose addition. When the applied cell voltage was increased to $0.8 \mathrm{~V}$, the cathode potential was approximately $-0.9 \mathrm{~V}$, and the $k$ and $\mathrm{E}_{\mathrm{r} 1 \mathrm{~h}}$ values increased to $1.202 \pm 0.124 \mathrm{~h}^{-1}$ and $70.60 \pm 4.21 \%$, respectively. However, the $k$ and $\mathrm{E}_{\mathrm{r} 1 \mathrm{~h}}$ values of the abiotic cathode control ( $0.8 \mathrm{~V}$ supply) were $0.825 \pm 0.024 \mathrm{~h}^{-1}$ and $53.82 \pm 1.80 \%$, respectively, and the corresponding values were $0.457 \pm 0.032 \mathrm{~h}^{-1}$ and $16.19 \pm 2.23 \%$ (Table 1 ) for the open circuit control. Therefore, compared to the open circuit and abiotic cathode controls, the $0.8 \mathrm{~V}$-supplied biocathode can significantly improve the reduction rate and efficiency of NFZ $(P \leq 0.03)$.

In order to exclude the additional effect of glucose on the biocathodic metabolism, the inorganic $\mathrm{NaHCO}_{3}$ worked as carbon source and electrode as electron donor were investigated. Similarly, with the increase of the applied voltage, $k$ and $\mathrm{E}_{\mathrm{r} 1 \mathrm{~h}}$ values accordingly increased. The NFZ degradation curve of the open circuit control did not accord with the apparent first-order kinetic equation, indicating that NFZ was difficult to be biodegraded without additional electron donor (e.g. glucose or electrode) (Fig. 2b). When the applied cell voltage was $0.2 \mathrm{~V}$, the $k$ and $\mathrm{E}_{\mathrm{r} 1 \mathrm{~h}}$ values were $0.597 \pm 0.084 \mathrm{~h}^{-1}$ and $46.62 \pm 4.95 \%$, respectively. When the applied cell voltage was $0.8 \mathrm{~V}$, the $k$ and $\mathrm{E}_{\mathrm{r} 1 \mathrm{~h}}$ values increased to $1.102 \pm 0.045 \mathrm{~h}^{-1}$ and $65.27 \pm 0.13 \%$, respectively. The $k$ and $\mathrm{E}_{\mathrm{r} 1 \mathrm{~h}}$ values of the open circuit control were $0.456 \pm 0.032 \mathrm{~h}^{-1}$ and $7.33 \pm 5.43 \%$, respectively (Table 1 ). The $k$ and $\mathrm{E}_{\mathrm{r} 1 \mathrm{~h}}$ values of the three biocathode modes $(0.2,0.5$ and $0.8 \mathrm{~V})$ were not significantly differed between the glucose and $\mathrm{NaHCO}_{3}$ performed groups $(P>0.05)$, in spite that $\mathrm{NaHCO}_{3}$ performed biocathodes had relatively lower $k$ and $\mathrm{E}_{\mathrm{r} 1 \mathrm{~h}}$ values. Importantly, compared to the abiotic cathode, the $\mathrm{NaHCO}_{3}$-fed biocathode still showed significant biocatalytic capacity $(P<0.01)$ 

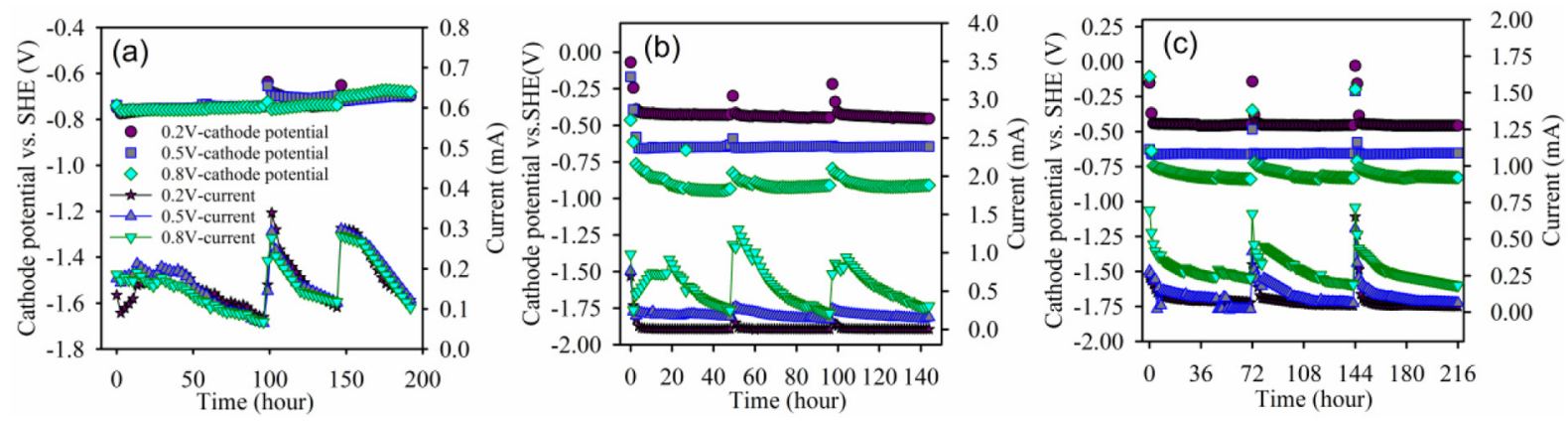

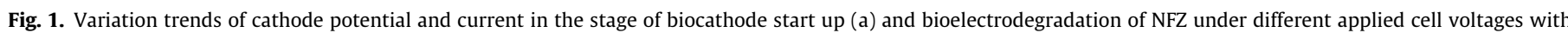
glucose (b) and $\mathrm{NaHCO}_{3}$ (c) as carbon sources.

Table 1

The $\mathrm{E}_{\mathrm{r} 1 \mathrm{~h}}$ and $k$ values of NFZ reduction under different conditions.

\begin{tabular}{|c|c|c|c|c|c|}
\hline & Applied voltage/V & Carbon source & $\mathrm{E}_{\mathrm{r} 1 \mathrm{~h}} / \%$ & $k\left(\mathrm{~h}^{-1}\right)$ & $r^{2}$ \\
\hline \multirow[t]{6}{*}{ Biocathode } & 0.2 & Glucose & $42.25 \pm 1.35$ & $0.677 \pm 0.069$ & $0.9906 \pm 0.0066$ \\
\hline & 0.2 & $\mathrm{NaHCO}_{3}$ & $46.62 \pm 4.95$ & $0.597 \pm 0.084$ & $0.9948 \pm 0.0025$ \\
\hline & 0.5 & glucose & $56.69 \pm 2.38$ & $0.913 \pm 0.049$ & $0.9930 \pm 0.0013$ \\
\hline & 0.5 & $\mathrm{NaHCO}_{3}$ & $53.44 \pm 1.39$ & $0.804 \pm 0.055$ & $0.9995 \pm 0.0004$ \\
\hline & 0.8 & Glucose & $70.60 \pm 4.21$ & $1.202 \pm 0.124$ & $0.9990 \pm 0.0008$ \\
\hline & 0.8 & $\mathrm{NaHCO}_{3}$ & $65.27 \pm 0.14$ & $1.102 \pm 0.045$ & $0.9997 \pm 0.0002$ \\
\hline Abiotic cathode & 0.8 & - & $53.82 \pm 1.80$ & $0.825 \pm 0.024$ & $0.9968 \pm 0.0012$ \\
\hline Open circuit & - & Glucose & $16.19 \pm 2.23$ & $0.456 \pm 0.032$ & $0.9648 \pm 0.0019$ \\
\hline
\end{tabular}

\subsection{Formation of NFZ reduction products under different operational conditions}

The degradation characterization and pathway of NFZ with the abiotic cathode under different cathode potentials had reported in our previous study. Though the degradation rate, efficiency and degree of NFZ were obviously affected by different cathode potentials, three mainly identified degradation products including $A M H$, AMN and NFF were consistent (Kong et al., 2015a). In this study, the three products of both the biocathode and open circuit biofilms were the same as previously identified based on the HPLC peak sequence and retention time.

The effect of different applied cell voltages on the formation of NFZ degradation products by the glucose-fed biocathodes was shown in Fig. 2. When NFF was accumulated to the maximum, it was further reduced to (5-amino-2-furan) methylamine (Fig. 2c). Importantly, the formation of NFF strongly suggests that the reduction of N-N and C-N bonds in NFZ could avoid the production of the high toxic biological metabolite of NFZ, semicarbazide (Kwon, 2017). Instead of semicarbazide, the organic nitrogen source urea could be generated during the NFZ degradation process, which may be used for the cathodophilic microbes. Moreover, the openloop reactions happened to produce straight chain hydrocarbons (5-hydroxycadaverine and 5-amino-pentanamide) at lower cathode potentials (Kong et al., 2015a), which may be acted as the potential organic carbon source for the cathodophilic microbes. In all the performed modes, AMH showed a tendency to increase first and then decrease (Fig. 2gh). Since it is a transition state of nitro to amine group of NFZ reduction, this intermediate can be rapidly reduced to the amine product, AMN. Apart from the $0.2 \mathrm{~V}$ supply condition, AMN showed the tendency to increase first and then decrease, which was further reduced and even open-loop happened (Fig. 2e). Additionally, the higher applied voltage resulted in the faster product generation rate or the subsequent degradation rate. When the applied voltage was $0.8 \mathrm{~V}$, the three products almost disappeared within $48 \mathrm{~h}$, indicating that the intermediate product was faster degraded by ring opening at a lower cathode potential. Importantly, the three products in the abiotic cathode BES were still markedly accumulated compared to the $0.8 \mathrm{~V}$-supply biocathode within $48 \mathrm{~h}(P<0.01)$. The NFF and AMN products increased gradually and accumulated in the open circuit biofilm, strongly indicating that the pure biodegradation process was difficult to destroy the heterocycle product to generate straight chain hydrocarbons (Fig. 2ce).

The effect of different applied voltages on the formation of NFZ degradation products by the $\mathrm{NaHCO}_{3}$-fed biocathodes was also shown in Fig. 2. When $0.2 \mathrm{~V}$ voltage was provided, the NFF and AMN products were obviously accumulated within $72 \mathrm{~h}$ (Fig. 2df), indicating that they were difficult to be further degraded under this condition. When applied with an increased voltage, the overall trends of the three products degradation dynamics were consistent with the glucose-fed biocathodes. Compared with the abiotic cathode, the biocathode still had a faster rate of product formation and further degradation at $0.8 \mathrm{~V}$. This indicated that the cathodophilic microbes had a relatively higher catalytic capacity even with $\mathrm{NaHCO}_{3}$ as carbon source and electrode as electron donor. The three products of the open circuit experiment were slowly accumulated. Combined with the glucose-fed open circuit experiment, we concluded that NFZ was difficult to be reduced because $\mathrm{NaHCO}_{3}$ only worked as carbon source but not electron donor (Wang et al., 2011). In addition, microbes could not get electrons from electrode with the circuit disconnected, and thus could not complete the normal microbial respiration. Collectively, the transformation of $\mathrm{NFZ}$ to the three main products maintained in the $\mathrm{NaHCO}_{3}$-fed biocathode although NFZ reduction rate and efficiency were relatively decreased compared to those of the glucose-fed biocathode. The degradation degree of the NFZ degradation products depended on the cathode potential but the composition of the NFZ degradation products were consistent at different operational conditions.

\subsection{CV, EIS, and SEM characterization}

The $\mathrm{CV}$ results showed that the biofilms had a similar feature and the onset potential was approximately $-0.35 \mathrm{~V}$ at 0.5 and $0.8 \mathrm{~V}$ applied voltages. The peak potential of NFZ reduction was approximately $-0.45,-0.60$ and $-0.75 \mathrm{~V}$ for $0.2,0.5$ and $0.8 \mathrm{~V}$ 

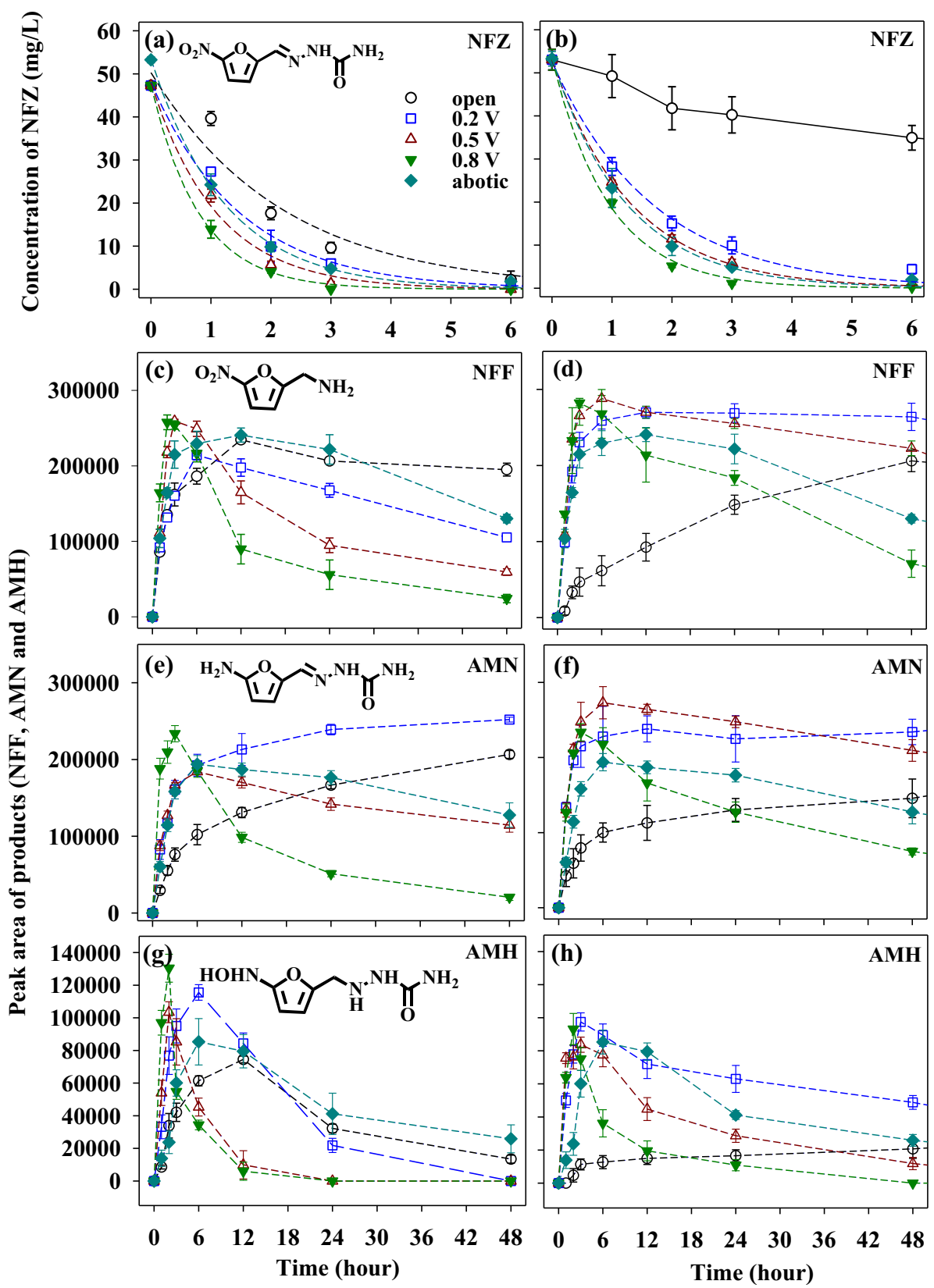

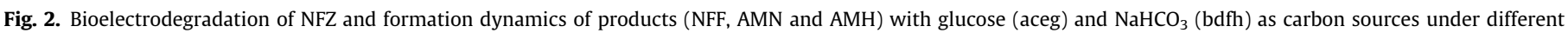
operational modes.

supply modes, respectively. The $0.2 \mathrm{~V}$ supply group also had a similar onset potential of $-0.35 \mathrm{~V}$, while it showed obviously higher peak current than the 0.5 and $0.8 \mathrm{~V}$ supply groups (Fig. 3a). This suggests that the biocathode with $0.2 \mathrm{~V}$ supply enriched more cathodophilic microbe, which likely resulted in the decreased over-potentials. Although the microbial catalytic abilities at 0.5 and $0.8 \mathrm{~V}$ were weaker than that at $0.2 \mathrm{~V}$, both of them were larger than that of the abiotic cathode. Additionally, the EIS results showed that the internal resistance of the cathodes at three applied cell voltages $(0.2,0.5$ and $0.8 \mathrm{~V})$ was $5.02,21.94$ and $23.15 \Omega$, respectively, which were significantly lower than that of the abiotic cathode (38.29 $\Omega$ ) (Fig. 3b). The CV and EIS results together strongly indicate that cathodophilic microbes enhanced the biocathodic electron transfer for the catalysis of NFZ. SEM results showed that the biofilms at open circuit, 0.2 and $0.5 \mathrm{~V}$ modes relatively enriched more cathodophilic microbes than that at $0.8 \mathrm{~V}$ mode, suggesting that the lower cathode potential and strong electrical stimulation could affect the formation of thick cathodic biofilm (Fig. S2).

\subsection{Overall microbial phylogenetic diversity}

Based on the Illumina high-throughput sequencing results, the number of $16 \mathrm{~S}$ rRNA gene sequences for different applied voltages $(0.2,0.5$ and $0.8 \mathrm{~V})$ and open circuit samples were 105624,56387 , 110194 and 142992, respectively (Table 2). Sufficient sequences strongly indicated the reliability of sequencing results. The Chao1 values from different cathodic biofilms were relatively higher than that of the open circuit biofilm. Other biodiversity indexes, such as $H, 1 / D$ and richness (OTU numbers after resample homogenization) also indicated the open circuit biofilm had the lowest phylogenetic diversity (Table 2). This maybe related to the fact that the source of 

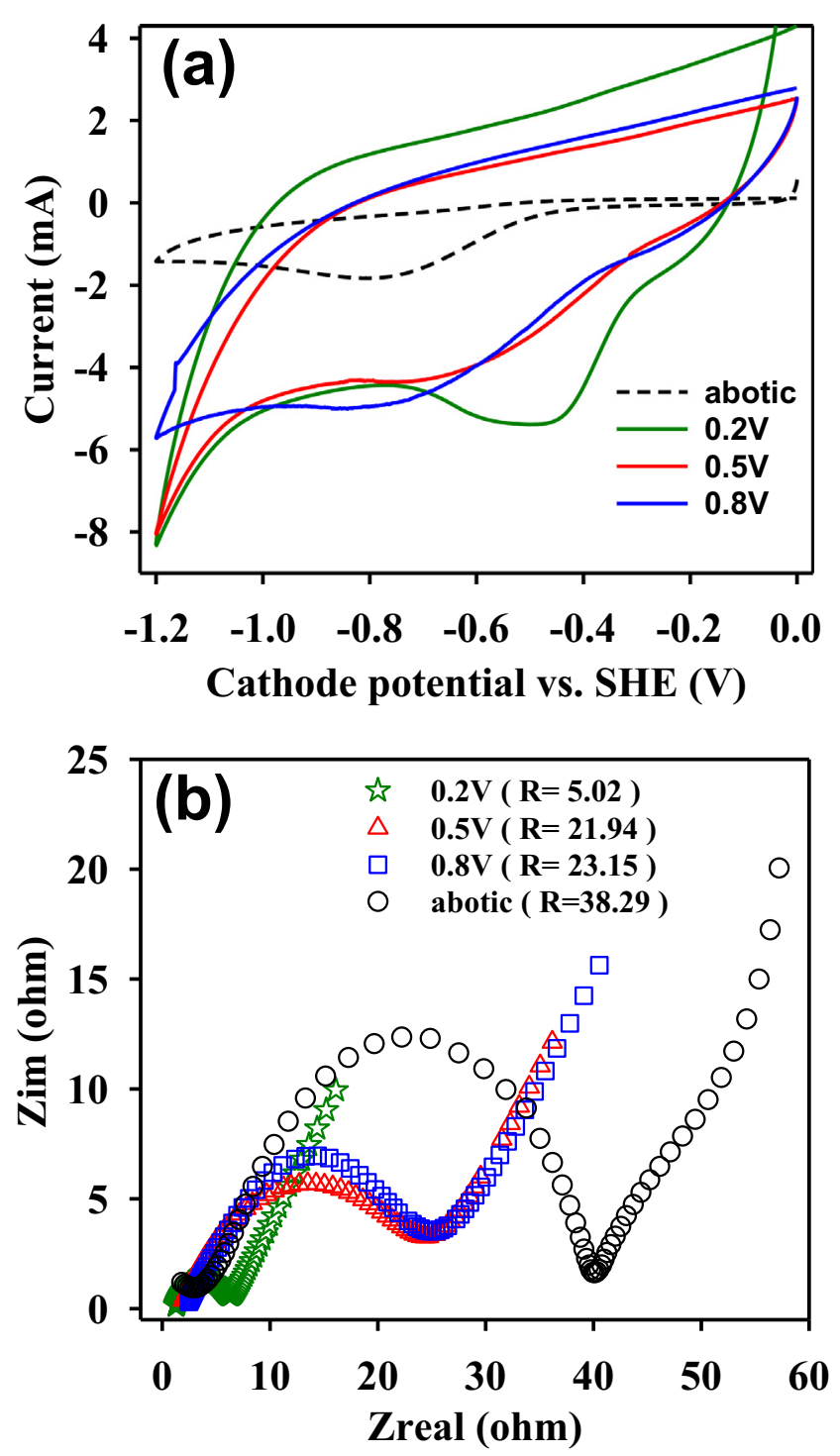

Fig. 3. Cyclic voltammograms (a) and nyquist plots (b) of three biocathodes and an abiotic cathode for NFZ reduction.

the electron donor in the open circuit biofilm was glucose, while electrode could be worked as the additional electron donor for cathodophilic bacteria in biocathode communities.

\subsection{Shifts in microbial community structure and composition}

Microbial community structures are similar for the three cathodic biofilms but are different from the open circuit biofilm based on the hierarchical clustering analysis of classified OTUs from each sample (Fig. 4a). Concretely, microbial community structures are highly similar for the 0.2 and $0.5 \mathrm{~V}$ applied cathodic biofilms. A total of 19 phyla were classified and most of sequences belonged to 5 phyla, including Proteobacteria, Bacteroidetes, Firmicutes, Syner- gistetes and Actinobacteria (Fig. 4b). The biofilms from 0.2 and $0.5 \mathrm{~V}$ supplied modes and open circuit control obviously enriched Proteobacteria, accounting for $84.81,75.55$ and $89.94 \%$, respectively. Interestingly, the lower cathode potential mode ( $0.8 \mathrm{~V}$ supply group) obviously enriched Firmicutes (accounting for 60.07\%), which was 4.07, 11.38 and 12.09 times higher than those of the $0.5 \mathrm{~V}$ group, $0.2 \mathrm{~V}$ group and open circuit control, respectively. A total of 45 classes were classified and most of sequences belonged to 9 classes (Fig. 4c). The biofilms from 0.2 and $0.5 \mathrm{~V}$ applied modes and open circuit control obviously enriched Gammaproteobacteria, accounting for $81.09,71.52$ and $88.23 \%$, respectively. The $0.8 \mathrm{~V}$ mode only had $11.85 \%$ of Gammaproteobacteria, while Bacilli and Actinobacteria dominated by 56.41 and $21.26 \%$, respectively. These clear differences strongly indicated that different cathode potentials shaped different bacteria communities. The lower cathode potential ( $0.8 \mathrm{~V}$ voltage supply) obviously led to a clear distinction compared to the 0.2 and $0.5 \mathrm{~V}$ performed biocathode communities.

\subsection{Potential function of dominant genera}

A total of 184 genera were classified among the four biofilm samples, of which 12 genera with relative abundance $>1 \%$ were shown in Fig. 5. Klebsiella was dominant in the $0.2 \mathrm{~V}$ (accounting for $62.54 \%$ ) and $0.5 \mathrm{~V}$ (accounting for $56.96 \%$ ) applied groups, while it was obviously decreased to $6.36 \%$ in the $0.8 \mathrm{~V}$ supply group. Previous studies have demonstrated that Klebsiella has nitroaromatics-reducing ability and electrochemical activity (Roldan et al., 2008; Xia et al., 2010). The $0.8 \mathrm{~V}$ supply group and open circuit control mainly enriched Enterococcus (accounting for 56.34\%) and Pseudomonas (accounting for $81.82 \%$ ), respectively. Previous studies have found that Enterococcus is often enriched in the nitroaromatic nitrobenzene-reducing biocathode communities, and the relative abundance can reach $38.4 \%$ (Liang et al., 2014) or 74.7\% (Wang et al., 2011). This was consistent with the fact that Enterococcus and Pseudomonas species own nitroaromaticsreducing ability (Rafii et al., 2003; Roldan et al., 2008). These results indicated that the microbial community structure with applied electrical stimulation was obviously different from that of the open circuit biofilm. Moreover, higher potential modes enriched a Gram-negative nitroaromatic reducer Klebsiella, and lower potential mode enriched a Gram-positive nitroaromatic reducer Enterococcus. Additionally, Citrobacter had relative abundances of $12.16 \%$ and $9.40 \%$ at $0.2 \mathrm{~V}$ and $0.5 \mathrm{~V}$ supply groups, respectively, while its relative abundances for the $0.8 \mathrm{~V}$ supply and open circuit modes were $0.61 \%$ and $5.80 \%$, respectively. Previous studies have proved that Citrobacter is an electroactive nitroaromatic reducer (Liang et al., 2017; Xu and Liu, 2011). A recent study has found that Citrobacter was obviously enriched by $29.24 \%$ in a glucose-fed azo dye-reducing biocathode community (Sun et al., 2016). Relatively low abundances of Desulfovibrio (from 1.63 to $2.04 \%$ in the electrode biofilms), Salmonella (from 0.14 to $1.17 \%$ in the electrode biofilms) and Clostridium $(4.35 \%$ in the open circuit biofilm) were also detected, which have been reported to carry out nitroaromatics reduction by nitroreductase (Roldan et al., 2008; Spain, 1995). Collectively, cathode potential regulation strategy stimulated the microbial electrode respiration activity, and enriched different electroactive nitroaromatic reducers (e.g. Klebsiella, Enterococcus, Citrobacter and Desulfovibrio)

Table 2

Microbial diversity indices of biocathode communities under different operational conditions.

\begin{tabular}{|c|c|c|c|c|c|c|}
\hline & No. of sequences & Chao1 & Richness & $H$ & $1 / D$ & Pielou evenness \\
\hline $0.2 \mathrm{~V}$ supply & 105624 & 378.38 & 304 & 1.98 & 2.92 & 0.35 \\
\hline $0.5 \mathrm{~V}$ supply & 56387 & 342.86 & 270 & 2.11 & 3.41 & 0.38 \\
\hline $0.8 \mathrm{~V}$ supply & 110194 & 446.13 & 359 & 1.90 & 3.07 & 0.32 \\
\hline Open circuit & 142992 & 313.97 & 222 & 1.32 & 1.96 & 0.24 \\
\hline
\end{tabular}



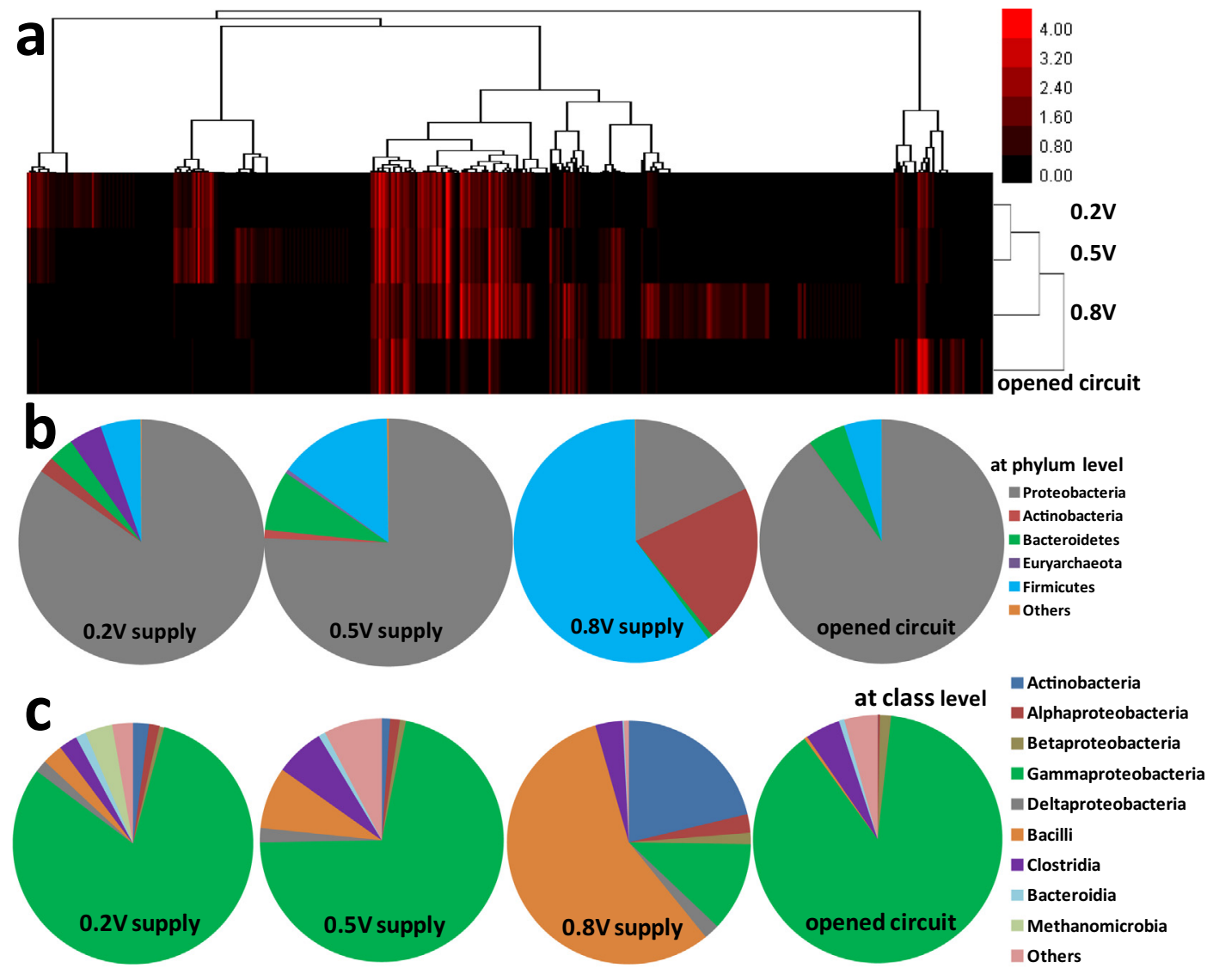

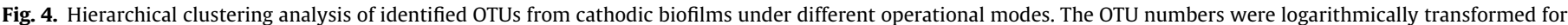

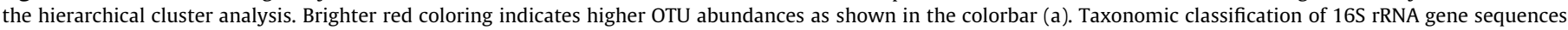

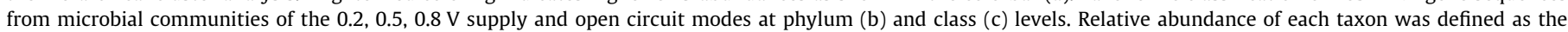

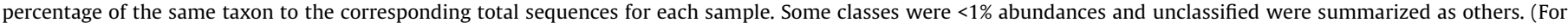
interpretation of the references to colour in this figure legend, the reader is referred to the web version of this article.)

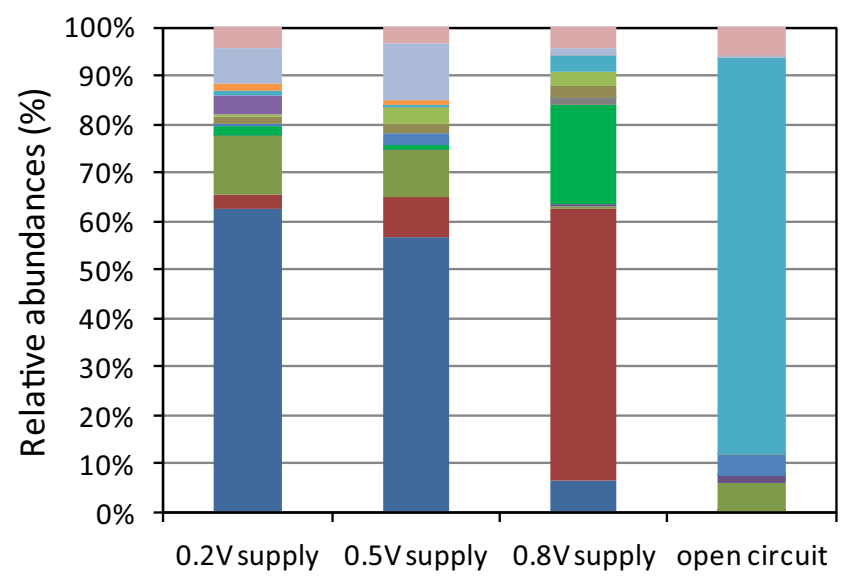

Others
Unclassified
Salmonella
Pseudomonas
Methanocorpusculum
Acetoanaerobium
Desulfovibrio
Clostridium XIVb
Brucella
Actinobaculum
Achromobacter
Citrobacter
Enterococcus
Klebsiella

Fig. 5. The dominant genera of biocathode communities under different operational modes.

respectively. These dominant functional genera likely played critical roles in enhancing the NFZ reduction and electrons transfer.

\section{Conclusions}

Ecological response of NFZ-degrading biocathode communities to different cathode potentials $(0.2,0.5$ and $0.8 \mathrm{~V}$ voltage groups) was investigated. Different cathode potentials obviously affected the bioelectrodegradation efficiency and degree of NFZ and shaped different biocathode communities. The 0.2 and $0.5 \mathrm{~V}$ performed biocathode communities were similar (both enriched a Gramnegative nitroaromatic reducer Klebsiella $>55 \%$ ) but significantly differed from the $0.8 \mathrm{~V}$ supply (enriched a Gram-positive nitroaromatic reducer Enterococcus by 56\%) and open circuit modes (enriched a Gram-negative nitroaromatic reducer Pseudomonas by $82 \%$ ). The dominant functional genera likely played critical roles in enhancing the NFZ catalysis and electrons transfer. 


\section{Acknowledgements}

This work was financially supported by the National Natural Science Foundation of China (No. 51508551 and 31500084), the Ministry of Environmental Protection of the People's Republic of China (Major Science and Technology Program for Water Pollution Control and Treatment) (Grant No. 2012ZX07202-005), and the Key Deployment Project of Chinese Academy of Sciences (No. ZDRW-ZS-2016-5-3).

\section{Appendix A. Supplementary data}

Supplementary data associated with this article can be found, in the online version, at http://dx.doi.org/10.1016/j.biortech.2017.06. 056.

\section{References}

Cheng, H.Y., Liang, B., Mu, Y., Cui, M.H., Li, K., Wu, W.M., Wang, A.J., 2015. Stimulation of oxygen to bioanode for energy recovery from recalcitrant organic matter aniline in microbial fuel cells (MFCs). Water Res. 81, 72-83.

Cui, D., Guo, Y.Q., Lee, H.S., Wu, W.M., Liang, B., Wang, A.J., Cheng, H.Y., 2014. Enhanced decolorization of azo dye in a small pilot-scale anaerobic baffled reactor coupled with biocatalyzed electrolysis system (ABR-BES): a design suitable for scaling-up. Bioresour. Technol. 163, 254-261.

Cui, M.H., Cui, D., Gao, L., Wang, A.J., Cheng, H.Y., 2016. Azo dye decolorization in an up-flow bioelectrochemical reactor with domestic wastewater as a costeffective yet highly efficient electron donor source. Water Res. 105, 520-526.

Feng, H., Zhang, X., Guo, K., Vaiopoulou, E., Shen, D., Long, Y., Yin, J., Wang, M., 2015. Electrical stimulation improves microbial salinity resistance and organofluorine removal in bioelectrochemical systems. Appl. Environ. Microbiol. 81 (11), 3737-3744.

Gao, S.H., Peng, L., Liu, Y.W., Zhou, X., Ni, B.J., Bond, P.L., Liang, B., Wang, A.J., 2016. Bioelectrochemical reduction of an azo dye by a Shewanella oneidensis MR-1 formed biocathode. Int. Biodeter. Biodegr. 115, 250-256.

Hong, Y., Lin, X., Cui, X., Zhou, L., Al-Rasheid, K.A.S., Li, J., 2015. Comparative evaluation of genotoxicity induced by nitrofurazone in two ciliated protozoa by detecting DNA strand breaks and DNA-protein crosslinks. Ecol. Indic. 54, 153160.

Jiang, X., Shen, J., Han, Y., Lou, S., Han, W., Sun, X., Li, J., Mu, Y., Wang, L., 2016a. Efficient nitro reduction and dechlorination of 2,4-dinitrochlorobenzene through the integration of bioelectrochemical system into upflow anaerobic sludge blanket: A comprehensive study. Water Res. 88, 257-265.

Jiang, X., Shen, J., Lou, S., Mu, Y., Wang, N., Han, W., Sun, X., Li, J., Wang, L., 2016b. Comprehensive comparison of bacterial communities in a membrane-free bioelectrochemical system for removing different mononitrophenols from wastewater. Bioresour. Technol. 216, 645-652.

Justino, C.I.L., Duarte, K.R., Freitas, A.C., Panteleitchouk, T.S.L., Duarte, A.C., RochaSantos, T.A.P., 2016. Contaminants in aquaculture: Overview of analytical techniques for their determination. TrAC Trend. Anal. Chem. 80, 293-310.

Kong, D., Liang, B., Lee, D.J., Wang, A., Ren, N., 2014. Effect of temperature switchover on the degradation of antibiotic chloramphenicol by biocathode bioelectrochemical system. J. Environ. Sci. 26 (8), 1689-1697.

Kong, D., Liang, B., Yun, H., Cheng, H., Ma, J., Cui, M., Wang, A., Ren, N., 2015a. Cathodic degradation of antibiotics: characterization and pathway analysis. Water Res. 72, 281-292.

Kong, D., Liang, B., Yun, H., Ma, J., Li, Z., Wang, A., Ren, N., 2015b. Electrochemical degradation of nitrofurans furazolidone by cathode: Characterization, pathway and antibacterial activity analysis. Chem. Eng. J. 262, 1244-1251.

Kwon, J.-W., 2017. Semicarbazide: Natural occurrence and uncertain evidence of its formation from food processing. Food Control 72 (Part B), 268-275.

Li, Y., Yang, H.Y., Shen, J.Y., Mu, Y., Yu, H.Q., 2016. Enhancement of azo dye decolourization in a MFC-MEC coupled system. Bioresour. Technol. 202, 93100.

Liang, B., Cheng, H., Van Nostrand, J.D., Ma, J., Yu, H., Kong, D., Liu, W., Ren, N., Wu, L., Wang, A., Lee, D.J., Zhou, J., 2014. Microbial community structure and function of nitrobenzene reduction biocathode in response to carbon source switchover. Water Res. 54, 137-148.

Liang, B., Cheng, H.Y., Kong, D.Y., Gao, S.H., Sun, F., Cui, D., Kong, F.Y., Zhou, A.J., Liu, W.Z., Ren, N.Q., Wu, W.M., Wang, A.J., Lee, D.J., 2013. Accelerated reduction of chlorinated nitroaromatic antibiotic chloramphenicol by biocathode. Environ. Sci. Technol. 47 (10), 5353-5361.

Liang, B., Kong, D., Ma, J., Wen, C., Yuan, T., Lee, D.J., Zhou, J., Wang, A., 2016. Low temperature acclimation with electrical stimulation enhance the biocathode functioning stability for antibiotics detoxification. Water Res. 100, 157-168.

Liang, S.-H., Hsu, D.-W., Lin, C.-Y., Kao, C.-M., Huang, D.-J., Chien, C.-C., Chen, S.-C., Tsai, I.J., Chen, C.-C., 2017. Enhancement of microbial 2,4,6-trinitrotoluene transformation with increased toxicity by exogenous nutrient amendment. Ecotox. Environ. Safe. 138, 39-46.

Pandey, P., Shinde, V.N., Deopurkar, R.L., Kale, S.P., Patil, S.A., Pant, D., 2016. Recent advances in the use of different substrates in microbial fuel cells toward wastewater treatment and simultaneous energy recovery. Appl. Energy 168, 706-723.

Pant, D., Van Bogaert, G., Diels, L., Vanbroekhoven, K., 2010. A review of the substrates used in microbial fuel cells (MFCs) for sustainable energy production. Bioresour. Technol. 101 (6), 1533-1543.

Qu, Y., Ma, Q., Deng, J., Shen, W., Zhang, X., He, Z., Van Nostrand, J.D., Zhou, J., Zhou J., 2015. Responses of microbial communities to single-walled carbon nanotubes in phenol wastewater treatment systems. Environ. Sci. Technol. 49 (7), 4627-4635.

Rafii, F., Wynne, R., Heinze, T.M., Paine, D.D., 2003. Mechanism of metronidazoleresistance by isolates of nitroreductase-producing Enterococcus gallinarum and Enterococcus casseliflavus from the human intestinal tract. FEMS Microbiol. Lett. 225 (2), 195-200.

Roldan, M.D., Perez-Reinado, E., Castillo, F., Moreno-Vivian, C., 2008. Reduction of polynitroaromatic compounds: the bacterial nitroreductases. FEMS Microbiol. Rev. 32 (3), 474-500.

Schloss, P.D., Westcott, S.L., Ryabin, T., Hall, J.R., Hartmann, M., Hollister, E.B., Lesniewski, R.A., Oakley, B.B., Parks, D.H., Robinson, C.J., Sahl, J.W., Stres, B., Thallinger, G.G., Van Horn, D.J., Weber, C.F., 2009. Introducing mothur: opensource, platform-independent, community-supported software for describing and comparing microbial communities. Appl. Environ. Microbiol. 75 (23), $7537-$ 7541.

Shen, J., Zhang, Y., Xu, X., Hua, C., Sun, X., Li, J., Mu, Y., Wang, L., 2013. Role of molecular structure on bioelectrochemical reduction of mononitrophenols from wastewater. Water Res. 47 (15), 5511-5519.

Spain, J.C., 1995. Biodegradation of nitroaromatic compounds. Annu. Rev. Microbiol. $49,523-555$.

Sun, F., Liu, H., Liang, B., Song, R., Yan, Q., Wang, A., 2013. Reductive degradation of chloramphenicol using bioelectrochemical system (BES): A comparative study of abiotic cathode and biocathode. Bioresour. Technol. 143, 699-702.

Sun, Q., Li, Z.L., Wang, Y.Z., Yang, C.X., Chung, J.S., Wang, A.J., 2016. Cathodic bacterial community structure applying the different co-substrates for reductive decolorization of Alizarin Yellow R. Bioresour. Technol. 208, 64-72.

Van Boeckel, T.P., Brower, C., Gilbert, M., Grenfell, B.T., Levin, S.A., Robinson, T.P., Teillant, A., Laxminarayan, R., 2015. Global trends in antimicrobial use in food animals. Proc. Natl. Acad. Sci. U.S.A. 112 (18), 5649-5654.

Vass, M., Hrusk, K., Franek, M., 2008. Nitrofuran antibiotics: a review on the application, prohibition and residual analysis. Vet. Med. Czech 53, 469-500.

Wang, A., Cheng, H., Ren, N., Cui, D., Lin, N., Wu, W., 2012. Sediment microbial fuel cell with floating biocathode for organic removal and energy recovery. Front. Environ. Sci. Eng. 6 (4), 569-574.

Wang, A.J., Cheng, H.Y., Liang, B., Ren, N.Q., Cui, D., Lin, N., Kim, B.H., Rabaey, K., 2011. Efficient reduction of nitrobenzene to aniline with a biocatalyzed cathode. Environ. Sci. Technol. 45 (23), 10186-10193.

Wang, H.M., Luo, H.P., Fallgren, P.H., Jin, S., Ren, Z.J., 2015. Bioelectrochemical system platform for sustainable environmental remediation and energy generation. Biotechnol. Adv. 33 (3-4), 317-334.

Wang, L., Liu, Y., Ma, J., Zhao, F., 2016. Rapid degradation of sulphamethoxazole and the further transformation of 3-amino-5-methylisoxazole in a microbial fuel cell. Water Res. 88, 322-328.

Xia, X., Cao, X.X., Liang, P., Huang, X., Yang, S.P., Zhao, G.G., 2010. Electricity generation from glucose by a Klebsiella sp. in microbial fuel cells. Appl. Microbiol. Biotechnol. 87 (1), 383-390.

Xu, S., Liu, H., 2011. New exoelectrogen Citrobacter sp. SX-1 isolated from a microbial fuel cell. J. Appl. Microbiol. 111 (5), 1108-1115.

Yu, W.-H., Chin, T.-S., Lai, H.-T., 2013. Detection of nitrofurans and their metabolites in pond water and sediments by liquid chromatography (LC)-photodiode array detection and LC-ion spray tandem mass spectrometry. Int. Biodeterior Biodegra. 85, 517-526.

Yun, H., Kong, D., Liang, B., Cui, M., Li, Z., Wang, A., 2016. Response of anodic bacterial community to the polarity inversion for chloramphenicol reduction. Bioresour. Technol. 221, 666-670.

Yun, H., Liang, B., Kong, D.Y., Cheng, H.Y., Li, Z.L., Gu, Y.B., Yin, H.Q., Wang, A.J. 2017a. Polarity inversion of bioanode for biocathodic reduction of aromatic pollutants. J. Hazard. Mater. 331, 280-288.

Yun, H., Liang, B., Qiu, J., Zhang, L., Zhao, Y., Jiang, J., Wang, A., 2017b. Functional characterization of a novel amidase involved in biotransformation of triclocarban and its dehalogenated congeners in Ochrobactrum sp. TCC-2. Environ. Sci. Technol. 51 (1), 291-300.

Zhang, Q., Zhang, Y., Li, D., 2017. Cometabolic degradation of chloramphenicol via a meta-cleavage pathway in a microbial fuel cell and its microbial community. Bioresour. Technol. 229, 104-110.

Zhang, Q.Q., Ying, G.G., Pan, C.G., Liu, Y.S., Zhao, J.L., 2015. Comprehensive evaluation of antibiotics emission and fate in the river basins of China: source analysis, multimedia modeling, and linkage to bacterial resistance. Environ. Sci. Technol 49 (11), 6772-6782.

Zhu, X., Yates, M.D., Hatzell, M.C., Ananda Rao, H., Saikaly, P.E., Logan, B.E., 2014 Microbial community composition is unaffected by anode potential. Environ. Sci. Technol. 48 (2), 1352-1358. 